\title{
Wide Swath, High Resolution SAR Using Multiple Receive Apertures
}

\author{
Nathan Goodman, Devindran Rajakrishna, Jim Stiles \\ The University of Kansas, Radar Systems and Remote Sensing Laboratory \\ 2291 Irving Hill Road, Lawrence, KS 66045 \\ Phone: (785) 864-7739, Fax: (785) 864-7789, E-mail: ngoodman@ rsl.ukans.edu
}

Abstract - When one attempts to image a large area while maintaining high resolution, ambiguities often appear. This occurs when the number of pixels exceeds the number of measurements or when the area includes range-Doppler ambiguities. In fact, the number of independent samples obtained by a single-receiver SAR, and consequently the number of pixels that can be unambiguously imaged, is equal to the time-bandwidth product of the radar signal. It is shown that multiple receive apertures resolve this scenario by adding independent angle-of-arrival samples to the system. Because targets that are ambiguous in range and Doppler are not ambiguous in angle of arrival, an image with the same resolution as the single-aperture image, but with larger area, is unambiguously obtained.

We present a multiple-aperture configuration, each with coherent receivers. The SAR image is calculated using three methods. The first is the traditional matched-filter method. The second is an orthogonal filter method. Last, a sidelobe cancellation filter is applied. The methods are applied after each aperture is range-Doppler processed. Therefore, the methods are used to resolve the ambiguities inherent in traditional range-Doppler processing.

\section{INTRODUCTION}

When SAR is implemented using a single receive aperture, the number of independent measurements is limited to the time-bandwidth product of the return radar signal. The resolution must be designed such that the number of pixels in the image does not exceed the time-bandwidth product and the swath width must not include range or Doppler ambiguities. This paper suggests using multiple apertures [1] each with its own coherent receiver. Each aperture adds independent samples to the radar system; furthermore, since the independent samples provide angle-of-arrival information, and since range-Doppler ambiguities will not be co-located, the range-Doppler ambiguities inherent in a single aperture can potentially be resolved. Resolving the range-Doppler ambiguities with angle information allows the swath width to be increased.

A signal space notation for the radar measurements and image reconstruction filters is presented. From this notation, the relation between the number of independent samples and the number of pixels that can be unambiguously imaged becomes clear. The signal space notation also exposes the difference between the three algorithms applied in this paper: matched filter, orthogonal filter, and sidelobe cancellation filter. Results are presented for a SAR with two receive apertures placed in closely spaced and sparse configurations.

\section{SIGNAL SPACE REPRESENTATION FOR SAR}

To facilitate the treatment of a multiple-receive-aperture SAR, the following signal space notation is developed for general treatment of a SAR system. The ith pixel will produce at the receive apertures a set of measurement samples in time and space. Those measurements form a response vector, $\mathbf{r}_{\mathbf{i}}$, in an $\mathbf{M}$-dimensional space where $\mathbf{M}$ is the total number of independent measurements. The total received signal seen by the radar is then the weighted sum of the responses from all targets,

$\mathbf{r}_{\text {meas }}=\sum \gamma_{i} \mathbf{r}_{i}$

where $\gamma_{i}$ is the complex rcs of the ith pixel. Fig. 1 shows a physical representation of this notation for a simple system of two targets and two independent samples. The matrix

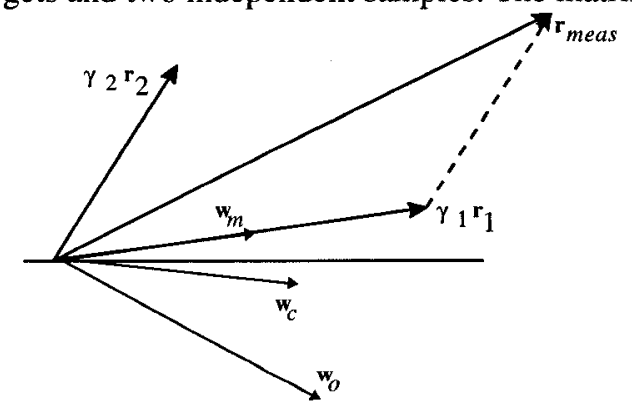

Fig 1: Target, Measurement, and Filter Vectors

representation of the radar's total received vector is

$\mathbf{r}_{\text {meas }}=\left[\begin{array}{llll}\mathbf{r}_{1} & \mathbf{r}_{2} & \cdots & \mathbf{r}_{N}\end{array}\right] \cdot\left[\begin{array}{c}\gamma_{i} \\ \gamma_{2} \\ \vdots \\ \gamma_{N}\end{array}\right]=\mathbf{R} \cdot \vec{\gamma}$

where the number of pixels is $\mathrm{N}$. We desire to obtain the vector of rcs values, $\vec{\gamma}$, but how well we can do so depends on the properties of the matrix $\mathbf{R}$. For $M$ measurements by the radar, $\mathbf{R}$ has dimension $\mathrm{M}$ by $\mathrm{N}$. We know that $\mathbf{M}$ must be greater than $\mathbf{N}$ or the columns in $\mathbf{R}$ will be linearly dependent. Furthermore, having enough measurements, $\mathbf{M}$, does not ensure linear independence. For a single-aperture SAR designed for a wide swath, range-Doppler ambiguities cause columns in $\mathbf{R}$ to be identical, at which point they cannot be distinguished. A multiple-receive-aperture SAR increases $\mathbf{M}$, the dimension of $\mathbf{R}$ 's columns, and the angle-of-arrival information gives the columns linear independence. 
From this representation, the physical meaning of the three SAR algorithms to be presented can be explained. The first algorithm is the traditional matched-filter approach. The matched filter for a given pixel is the response vector for that pixel. The matched-filter vector for pixel one is shown as $\mathbf{w}_{m}$ in Fig. 1. The estimate for pixel one is the inner product between $\mathbf{w}_{\mathrm{m}}$ and the measurement vector. Since $\mathbf{r}_{1}$ and its weight vector are aligned, this approach gives the most signal power from the pixel of interest. The weight vector, however, is not orthogonal to $\mathbf{r}_{2}$. Since $\mathbf{r}_{2}$ contributes to the total response measured by the radar, a portion of the energy received from pixel two will appear in the estimate of the rcs of pixel one.

In order to prevent energy from undesired pixels from appearing in the estimate of the desired pixel, an orthogonal filter can be used. The orthogonal filter for estimating pixel one is shown as $\mathbf{w}_{\mathbf{0}}$ in Fig. 1. Only energy from pixel one will be picked up by $\mathbf{w}_{0}$. A problem occurs when $\mathbf{r}_{1}$ and $\mathbf{r}_{2}$ are closely aligned, or highly ambiguous. The vector chosen orthogonal to $\mathbf{r}_{2}$ will be almost orthogonal to $\mathbf{r}_{1}$, and small amounts of noise will cause large variations in the estimate of pixel one.

A third method is a compromise between the matched filter and orthogonal filter. The method begins with the matched filter. An additional component chosen orthogonal to the matched filter is added such that the final filter has components parallel and orthogonal to the matched filter. The orthogonal component minimizes the total output power from the filter while the parallel component ensures that enough energy is obtained from the desired pixel. This sidelobe cancellation filter will lie somewhere between the matched and orthogonal filters as shown by $\mathbf{w}_{\mathrm{c}}$ in Fig. 1. This method is taken from the generalized sidelobe canceller presented in [2].

\section{SIMULATIONS}

This section presents results obtained through simulation of a SAR. Fig. 2 shows the original test pattern. Fig. 3 shows the poor results obtained when a single-aperture SAR illuminates more pixels than the time-bandwidth product can handle.

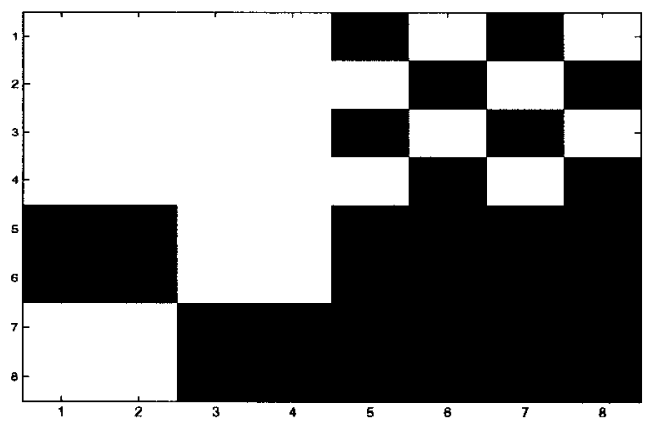

Fig 2: Original Test Pattern For SAR Simulations

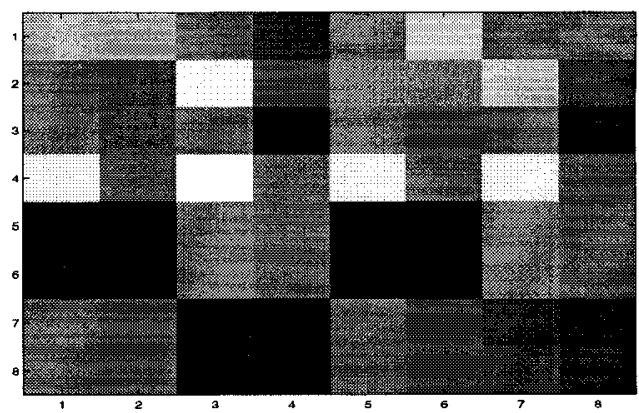

Fig 3: Single-Receiver SAR, Insufficient Time-Bandwidth

Next, we demonstrate the advantage of adding a second receive aperture. The total number of independent samples is 64 , as is the number of pixels in the test pattern. The second aperture is placed such that the Doppler ambiguities lie in the array pattern null. The matched-filter result is shown in Fig. 4 and can be thought of as the simultaneous application of conventional range-Doppler processing and conventional beamforming. Pixels that are ambiguous in Doppler are not ambiguous in angle of arrival, and a good image results.

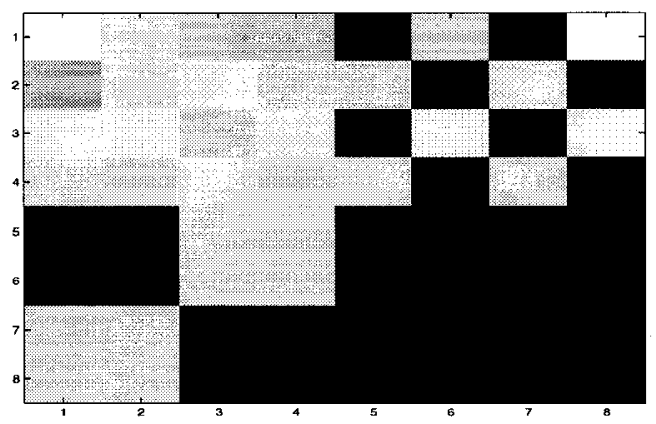

Fig 4: Two-Receiver SAR, Matched Filter

The decreased ambiguity resulting from the addition of a second receive aperture can be demonstrated another way. Two ambiguous targets will have closely aligned response vectors, and their inner product will be large. The amount of ambiguity between all combinations of pixels is easily calculated by pre-multiplying $\mathbf{R}$ by its conjugate transpose. The resulting correlations for a single aperture and multiple receive apertures are shown in Fig. 5 and Fig. 6, respectively.

In Figs. 5 and 6, the intersection of the ith row and jth column is the correlation between the ith and jth pixels for the given radar design. The main diagonal is the correlation of each pixel with itself. We desire the main diagonal to be as bright as possible while making the off-diagonal elements dark, or zero. Fig. 6 demonstrates that the addition of a second receive aperture helps in reaching that goal. Each row or column also makes up the ambiguity function for the pixel that is constant in that row or column. Therefore, having zero off-diagonal elements corresponds to the ideal thumbtack ambiguity function. 


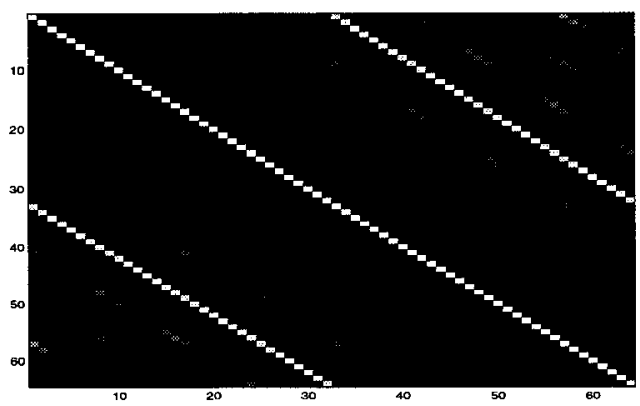

Fig 5: Correlation Matrix for Single-Receiver SAR

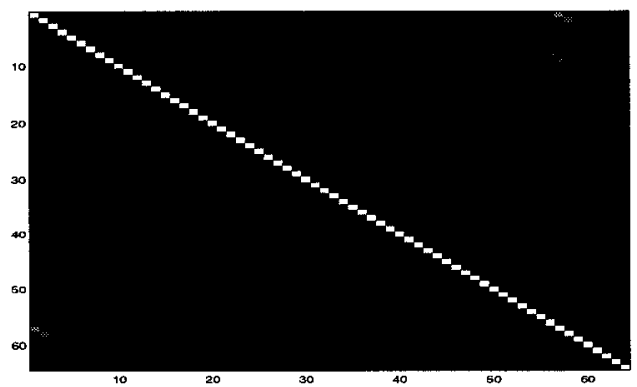

Fig 6: Correlation Matrix for Two-Receiver SAR

The previous results do not accurately reflect the amount of ambiguity or correlation in systems where multiple free-flying apertures cannot be placed close enough to avoid angle-ofarrival ambiguities. Fig. 7 demonstrates what can happen when the matched filter is applied to sparsely spaced receive apertures. Although none of the pixels are exactly ambiguous, sidelobes in range, Doppler, and angle of arrival

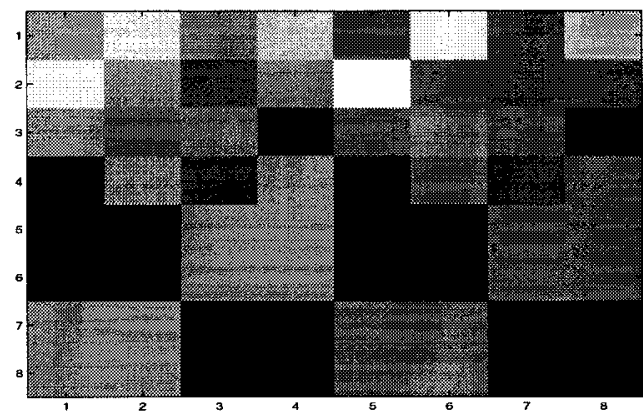

Fig 7: Two-Receiver SAR, Sparse, Matched Filter

cause high correlations. Consequently, we must try algorithms that minimize undesired contributions from correlated pixels. Fig. 8 demonstrates the results from matched-filter range-Doppler processing in association with a filter that is orthogonal to all Doppler ambiguities in angle of arrival. Considering the SNR issues concerning the orthogonal filter, it is noted here that the simulation has no noise.

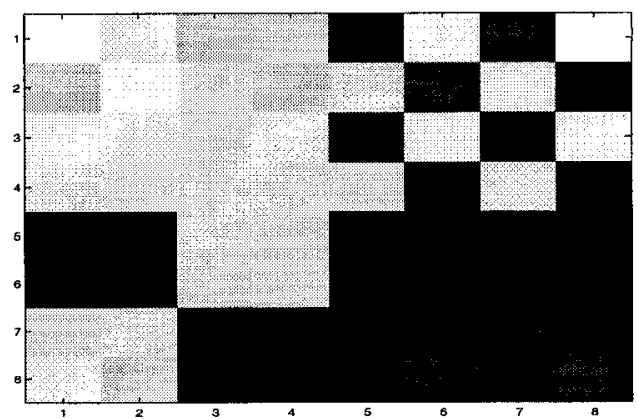

Fig 8: Two-Receiver SAR, Sparse, Orthogonal Filter

In reality, noise may cause inaccurate results using the orthogonal filter. The sidelobe cancellation method minimizes the contribution from Doppler ambiguities while constraining the SNR to an acceptable level. The result from the same simulation using the sidelobe cancellation filter is shown in Fig. 9.

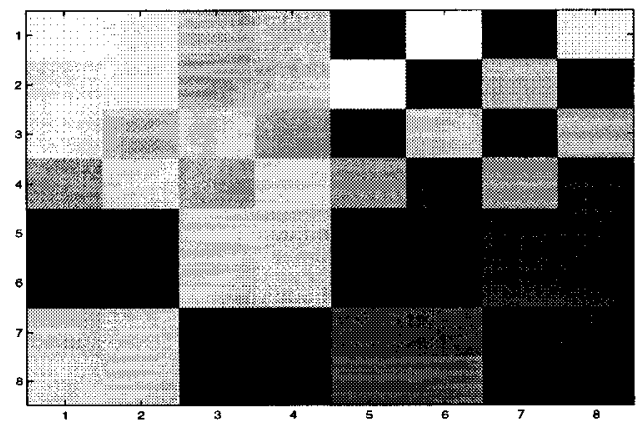

Fig 9: Two-Receiver SAR, Sparse, Sidelobe Cancellation

\section{CONCLUSIONS}

To accomplish wide swath, high resolution SAR, the number of independent radar samples must be increased and angle-of-arrival information must be obtained. It was shown that multiple apertures with coherent receivers accomplish both requirements. A signal space representation of the radar was presented and facilitated the discussion of reconstruction filter algorithms. Correlations between target responses were presented to show decreased ambiguity resulting from angleof-arrival information. Results were also presented that demonstrate the feasibility of wide swath, high resolution SAR in a sparse aperture configuration.

\section{REFERENCES}

[1] S.A. Hovanessian, L.B. Jocic, J.M. Lopez, "Spaceborne radar design equations and concepts," 1997 IEEE Aerospace Conf., vol. 1, pp. 125-136, Feb., 1997.

[2] D.H. Johnson and D.E. Dudgeon, Array Signal Processing, Prentice-Hall, New Jersey, 1993. 